Supporting Information

\title{
Atomically architected Si pyramid single-crystalline structure supporting epitaxial material growth and characteristic magnetism
}

\author{
Aydar Irmikimov ${ }^{1}$, Liliany N. Pamasi ${ }^{1}$, Azusa N. Hattori ${ }^{2}$, Takaaki Higashi ${ }^{1}$, Shunta Takahashi ${ }^{1}$, \\ Emilia E. Hashamova ${ }^{1,3}$, Xiaoqian $\mathrm{Shi}^{4}$, Fangzhun $\mathrm{Guo}^{4}$, Nobuyoshi Hosoito ${ }^{1}$, Ai I. Osaka ${ }^{2}$, Hidekazu \\ Tanaka $^{2}$, and Ken Hattori ${ }^{1, *}$
}

${ }^{1}$ Graduate School of Science and Technology, Nara Institute of Science and Technology (NAIST), Takayama 8916-5, Ikoma, Nara 630-0192, Japan

${ }^{2}$ Institute of Scientific and Industrial Research, Osaka University, Mihogaoka 8-1, Ibaraki, Osaka 5670047, Japan

${ }^{3}$ Department of Engineering, Karlsruhe Institute of Technology, Kaiserstraße 12, Karlsruhe 76131, Germany

${ }^{4}$ Institute of Mechanical Engineering, Dalian Jiaotong University, Huanghe Road 794, Dalian, Liaoning 116028, China

*E-mail: khattori@ms.naist.jp

\section{Fabrication of Si pyramid structure}

Si pyramid samples were prepared by photolithography and dry RIE, followed by wet chemical etching. SEM images of the samples after the treatments are shown in Figure S1. After the spin coating of the photoresist on $\mathrm{Si}(001)$ substrates, a square mask pattern of $16 \times 16 \mu \mathrm{m}^{2}$ area with $3 \mu \mathrm{m}$ space width was drawn by photolithography, where the square edge direction was $\langle 110\rangle$. Then, $50 \mathrm{~nm} \mathrm{Cr}$ was deposited on the mask pattern. By dipping the samples in acetone, the $\mathrm{Cr}$ film on the resist was removed and a square $\mathrm{Cr}$ mask pattern remained (Figure S1a). RIE induced the removal of the Si substrate in regions depending on the $\mathrm{Cr}$ mask shape; a square $\mathrm{Cr}$ mask with a certain $\mathrm{Si}$ crystalline direction leads to Si pyramid structures (Figure S1b). The Cr mask was removed by ultrasonication in acetone after RIE (Figure S1c), however, the pyramid surfaces were neither smooth nor oriented. Then, the samples were chemically etched under the optimized conditions, resulting in the smooth pyramid surfaces shown in Figure 1.

It is worth to mention the controllability of the size and distribution of pyramids. The minimum size is restricted by the wavelength dominantly in the photolithography. In our case, $\sim 1-2 \mu \mathrm{m}$ for $\mathrm{Hg}$ 
g-line $(\lambda=436 \mathrm{~nm})$. When we use electron beam lithography, instead of photolithography, we can reduce the size to sub $10 \mathrm{~nm}$ scale. The distribution is determined by the mask pattern; there is a competition between area-space ratio and RIE conditions. For instance, too narrow space hardly induces the etching initiated from the space. In contrast, too wide space induces small pyramid area and roughness of the substrate. Here we used one of the optimal conditions as an area of $16 \times 16 \mu \mathrm{m}^{2}$ and a space of $3 \mu \mathrm{m}$.
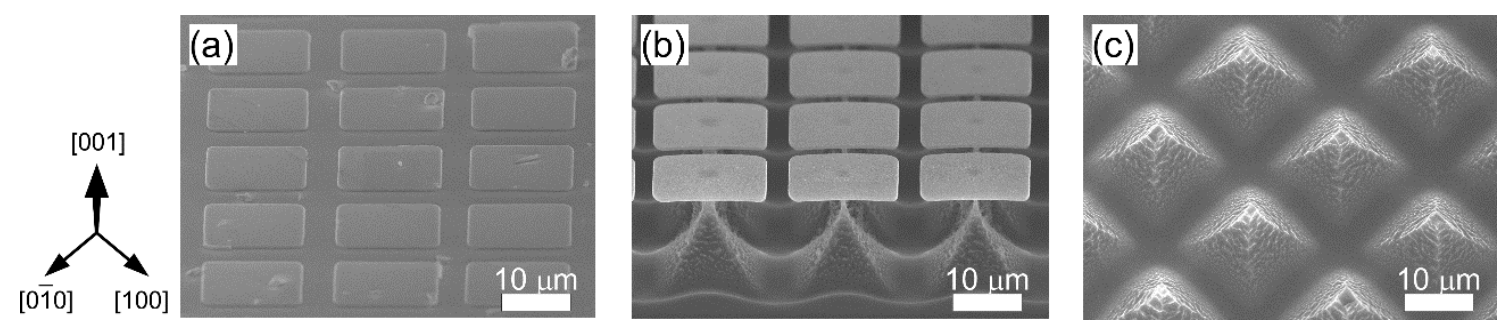

Figure S1. SEM images taken during the fabrication processes for the Si pyramid structure: (a) square Cr mask pattern on a $\mathrm{Si}(001)$ substrate, (b) dry-etched $\mathrm{Si}$ substrate with the Cr masks after RIE, and (c) Si pyramids after the removal of the Cr masks.

\section{LEED patterns of $\operatorname{Si}\{111\}$ pyramid surfaces fabricated on a $\operatorname{Si}(001)$ substrate.}

The Ewald diffraction conditions in LEED for the pyramid sample, which correspond to the simulated LEED patterns in Figure 3, are explained using the cross section of the 3D reciprocal space map in Figure S2. Symbol $O$ denotes the origin of the reciprocal space. Reciprocal lattice rod $00_{\text {planar }}$ is perpendicular to the planar $\mathrm{Si}(001)$ substrate plane passing through origin $O$. The other fundamental reciprocal lattice rods such as $01_{\text {planar }}$ are perpendicular to the substrate plane. Under the Ewald conditions, the crossing points (e.g., points $P$ and $Q$ ) of the reciprocal lattice rods (black lines) with the Ewald sphere (orange circle) corresponds to the diffraction points, the projection of which on the screen appears as diffraction spots (e.g., spots $P^{\prime}$ and $Q^{\prime}$ ) in LEED. Wavenumber vectors $\overrightarrow{S O}$ and $\overrightarrow{S P}(\overrightarrow{S Q})$ correspond to the incident and diffraction electrons, respectively. When the Ewald sphere radius $(|\overrightarrow{S O}|)$ increases with $E_{p}$, the diffraction spots (e.g., $\left.Q^{\prime}\right)$ from the substrate plane move to the screen center $\left(P^{\prime}\right)$, because angle $\angle P S Q$ decreases while acceptance angle $\theta_{a}$ is fixed by the screen size and the distance between the sample and LEED optics.

A well-ordered facet-plane surface also leads to reciprocal lattice rods (e.g., $00_{\text {facet }}$ ) perpendicular to the facet plane, shown as green lines, but the rod direction is tilted from the substrate normal. With increasing $E_{p}$, diffraction spots from the facet plane (e.g., $R^{\prime}$ ) do not move to the screen center but to the point of $00_{\text {facet }}$ crossing the Ewald sphere. Figure 2 illustrates a 3D schematic of the crossing between the facet reciprocal lattice rods and the Ewald sphere displaying diffraction spots on the screen; blue spots from one of the four equivalent $\operatorname{Si}\{111\} 1 \times 1$ facet surfaces are shown. Here,

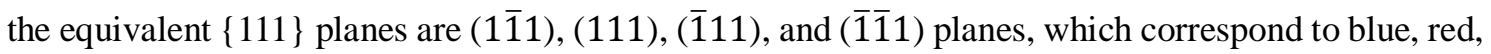


yellow, and green diffraction spots, respectively.

Figures $3 \mathrm{c}$ and $3 \mathrm{~d}$ illustrate diffraction spots from the four equivalent facet surfaces in blue, red, yellow, and green at different $E_{p}=56$ and $64 \mathrm{eV}$, respectively. The green and red spots reflect clean $\operatorname{Si}\{111\} 7 \times 7$ and $\operatorname{Si}\{111\} 2 \times 2-F e$ reconstructions, respectively. With increasing $E_{p}$, the facet spots move in the four outward directions determined by $00_{\text {facet }}$ rods, while the substrate $\mathrm{Si}(001) 1 \times 1$ spots move to the screen center. For the clean surfaces (Figure $3 \mathrm{a}, \mathrm{b}$ ), substrate clean $\mathrm{Si}(001) 2 \times 1 / 1 \times 2$ reconstructed spots were also seen. Such motion was confirmed by the experimental $\operatorname{Si}\{111\} 7 \times 7$ and $2 \times 2$-Fe patterns with the $1 \times 1$ simulation patterns on the LEED screen as a function of $E_{p} \quad(=50-140$ eV) in the separately uploaded video file "LEED_clean7x7_sim_2x2-Fe.avi" and also in Figure S3. Note that the intensities of the reconstructed spots have an $E_{p}$ dependence as those on general planar surfaces.

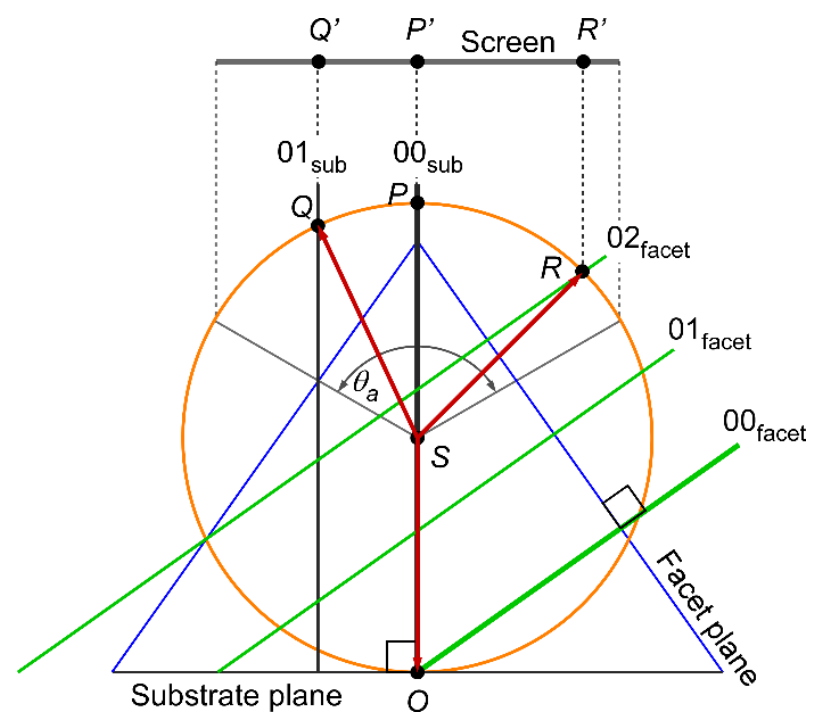

Figure S2. Schematic of the Ewald diffraction conditions in LEED for the pyramid sample. The black and green lines are reciprocal lattice rods from the substrate and facet planes, respectively. The orange circle is the Ewald sphere. Symbols $O, S, P$, and $R$ denote the origin of the reciprocal space, the Ewald sphere center, and the crossing points of the reciprocal lattice rods with the Ewald sphere. Wavenumber vectors such as $\overrightarrow{S P}, \overrightarrow{S Q}$, and $\overrightarrow{S R}$ correspond to diffraction electrons, the projection of which on the screen, within acceptance angle $\theta_{a}$, appear as diffraction spots (e.g., $P^{\prime}, Q^{\prime}$, and $R^{\prime}$ ). 

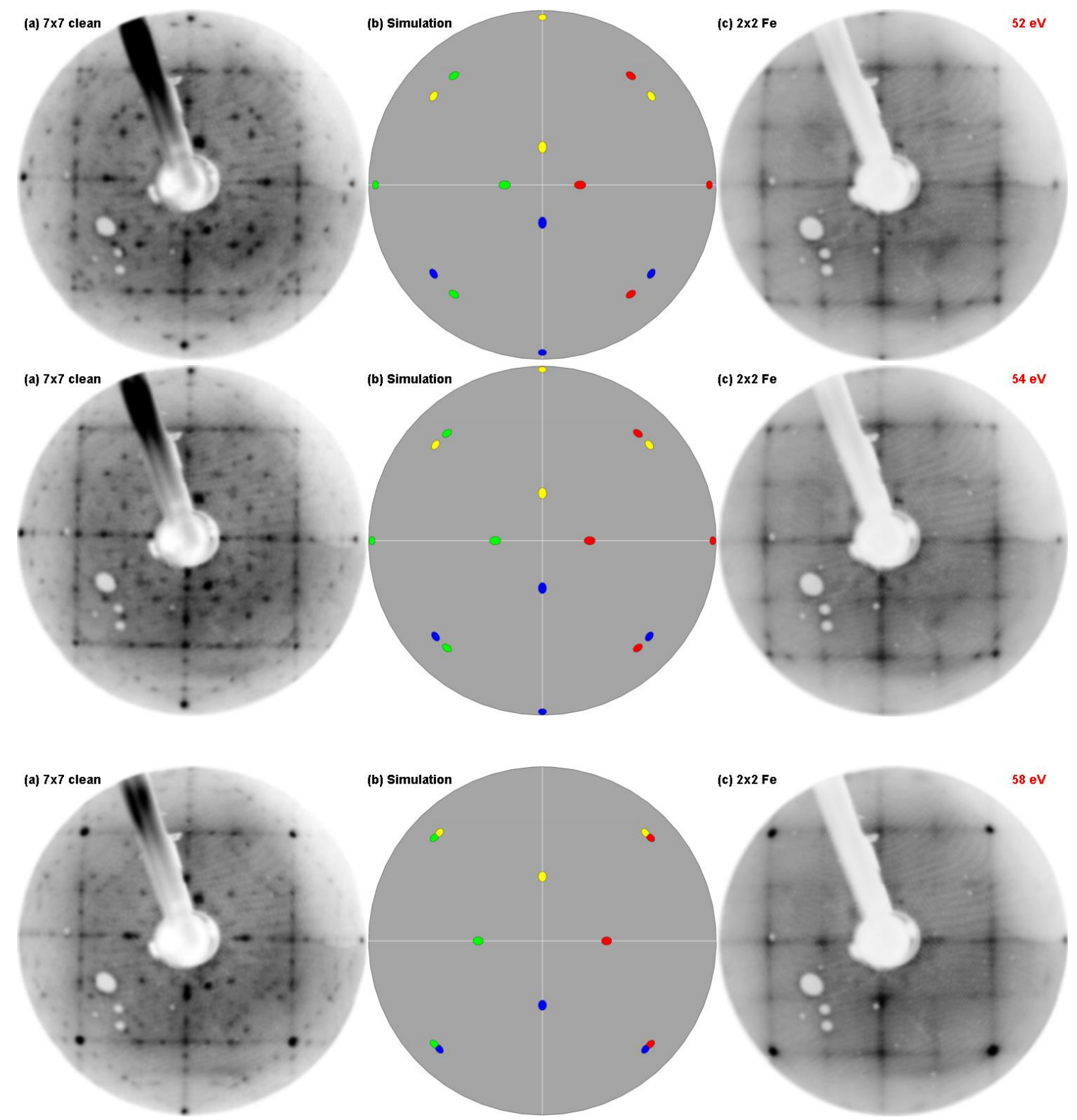

(c) $2 \times 2 \mathrm{Fe}$

$58 \mathrm{eV}$
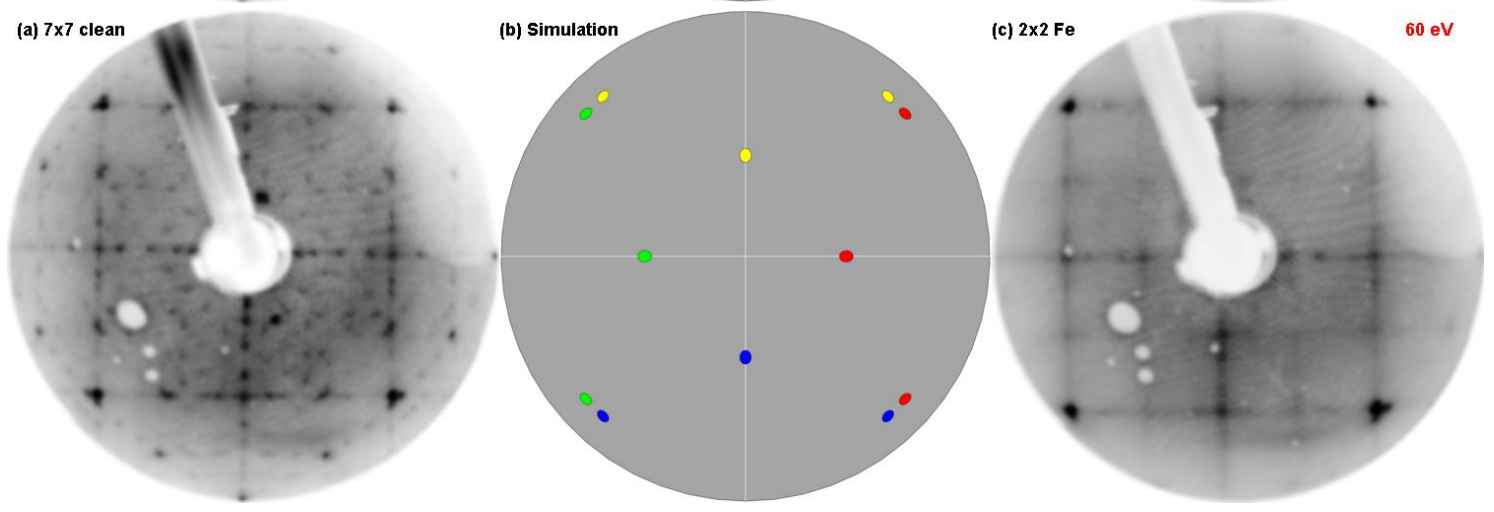


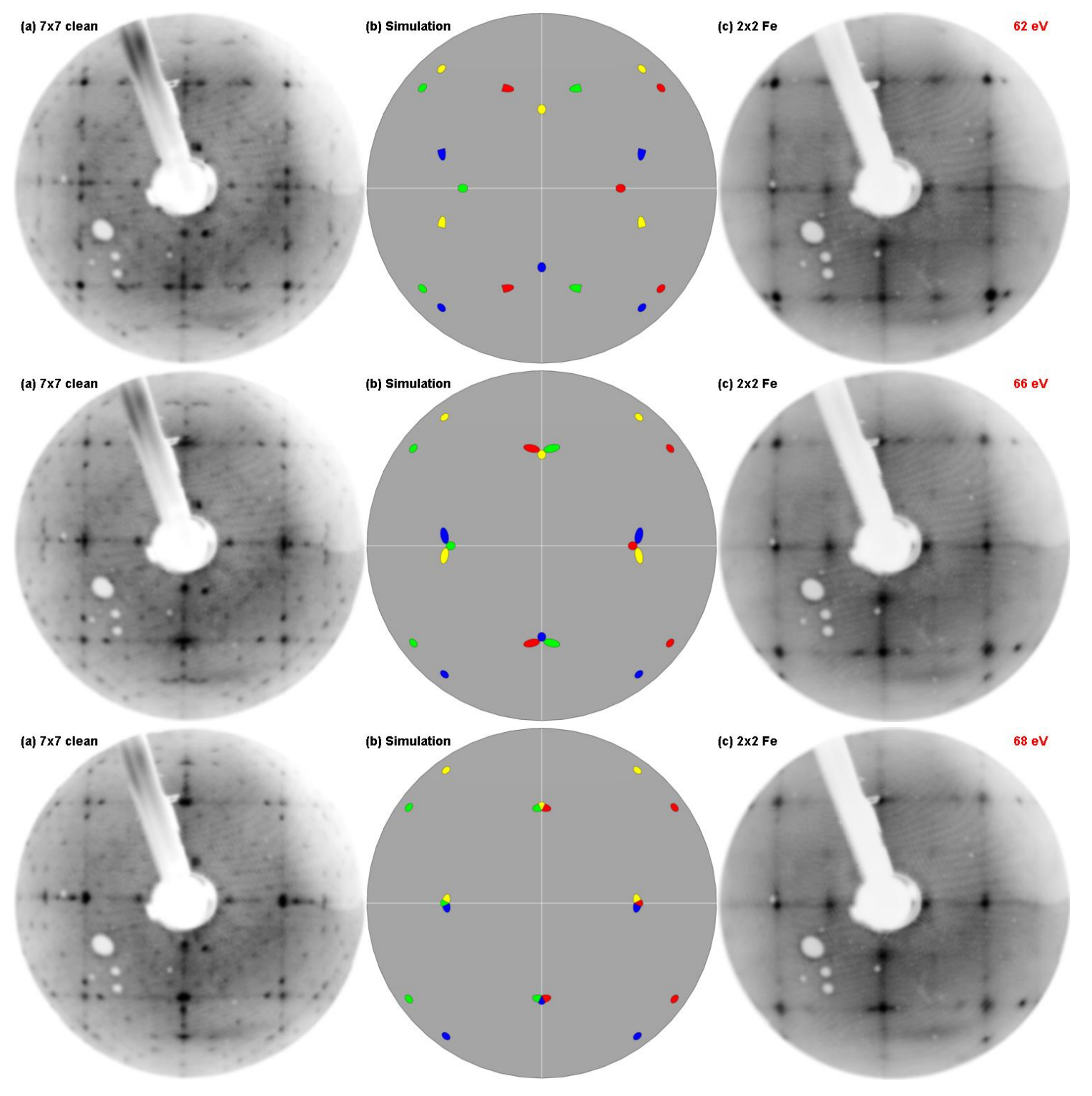

Figure S3. $E_{p}$-dependent LEED patterns of clean $\operatorname{Si}\{111\} 7 \times 7$ (left panel), simulation (central part), and $\mathrm{Si}\{111\} 2 \times 2-\mathrm{Fe}$ (right panel) from four equivalent facet surfaces of the Si pyramid; spots from each facet surface are identified by blue, red, yellow, and green colors.

\section{TEM observation}

Middle-scaled TEM images between Figures $4 a$ and $4 c$ are shown in Figure S4. In Figure S4a, a line-like structure (labeled by red lines) corresponding to an Fe nanofilm can be seen on a Si facet surface capped with a TEM glue. The dark bands near the Si edge are caused by the interference effect along $\mathrm{Si}[1 \overline{1} 1]$ in the TEM milled sample; note that the thickness of this wedge-shaped region was $<50$ $\mathrm{nm}$. The magnified image in Figure S4b clearly displays the atomic structure of bcc-Fe on the Si facet 
surface, $\mathrm{Fe}(111)[11 \overline{2}] \quad \| \operatorname{Si}(111)\langle\overline{11} 2\rangle$, as indicated in Figure 4c. Some interface regions were tilted from the $\mathrm{Si}\{111\}$ plane (the $\{111\}$ plane is indicated by red lines) owing to a fluctuation.

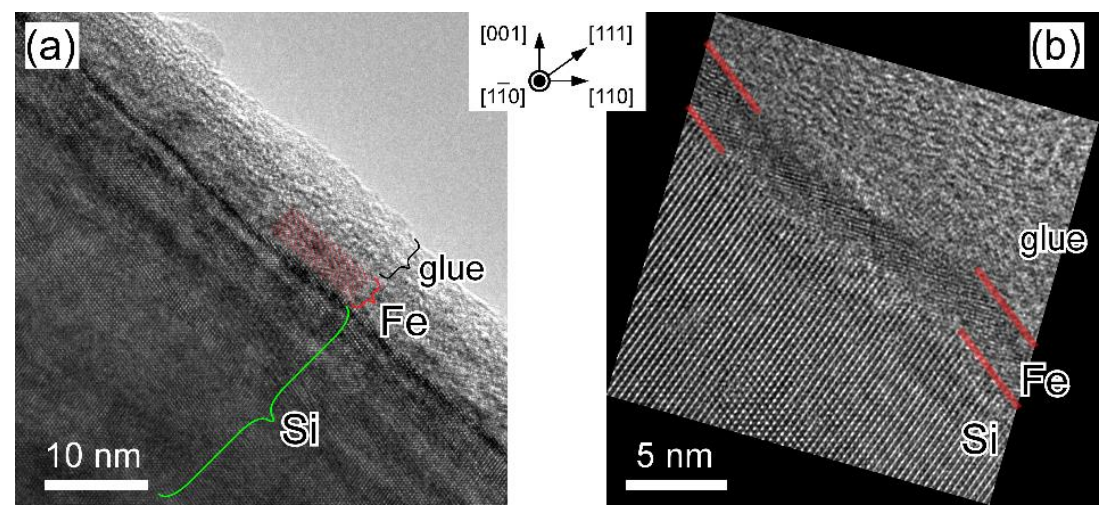

Figure. S4. TEM images of the $\mathrm{Si}\{111\}$ pyramid sample deposited with $\mathrm{Fe}(2 \mathrm{~nm})$ in facet surface regions. Both (a) and (b) show an epitaxially grown bcc-Fe film on the Si facet surfaces.

\section{Magnetism}

Micromagnetic states for pyramidal ferromagnetic films have been simulated in References [1-3]. Reference [1] classified micromagnetic configurations into seven different states: single domain, onion, flower, S, C, symmetric vortex, and asymmetric vortex states, as a function of film thickness and pyramid edge length. In the phase diagram of the magnetism in pyramidal structures, our pyramid $\left(l=16 \mu \mathrm{m}\right.$ and $\left.\Theta_{\mathrm{Fe}}=30 \mathrm{~nm}\right)$ corresponds to the asymmetric vortex ground state for remanence, as shown in Figure 5c (point 2).

For the change in the magnetization by the out-of-substrate-plane magnetic field, we can predict simple schematics in Figure S5, from the simulations [1-3]. The figure explains that, at point $\mathbf{1}$ under the higher negative magnetic field, the magnetization is aligned in the downward direction, and changes to cant like a screw with decreasing the field, for instance, at remanence (2). With increasing the field in the upper direction, the magnetization passes an in-plane vortex state $(\mathbf{3})$ and is aligned in the upward direction at the higher positive field (4). Note that out-of-plane magnetization directions (for the substrate and facet planes) are hard axes (1, 2 and 4), as shown in the inset in Figure 5b and References [4-6]. Thus, a magnetization has a tendency to lie in in-plain directions at weak fields (2 and 3). 


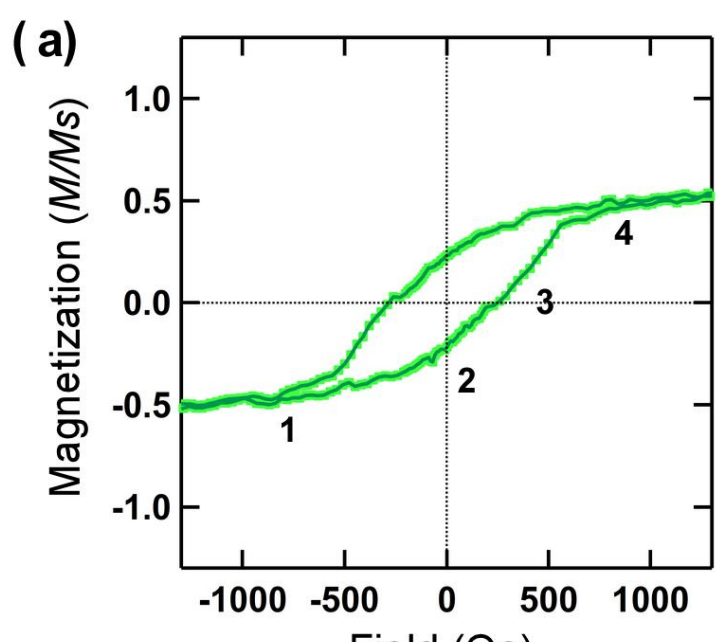

(b)
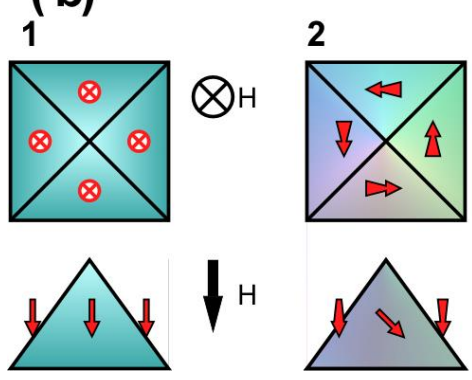

Field $(\mathrm{Oe})$

Figure S5. (a) Magnetization as a function of magnetic field in out-of-substrate-plane direction (Figure $5 b$ ), and (b) schematic illustrations of the magnetization in each point.

\section{References}

[1] A. Knittel, M. Franchin, T. Fischbacher, F. Nasirpouri, S.J. Bending, and H. Fangohr, New J. Phys. 12, 113048 (2010).

[2] F. Nasirpouri, M.A. Engbarth, S.J. Bending, L.M. Peter, A. Knittel, H. Fangohr, and M.V. Milosevic, Appl. Phys. Lett. 98, 222506 (2011).

[3] A. Knittel, M. Franchin, F. Nasirpouri, S.J. Bending, and H. Fangohr, J. Appl. Phys. 111, 07D127 (2012).

[4] Z.H. Nazir, C.-K. Lo, and M. Hardiman, J. Magn. Magn. Mater. 156, 435 (1996).

[5] A.N. Hattori, K. Hattori, and H. Daimon, J. Phys. Conf. Ser. 61, 404 (2007).

[6] A.N. Hattori, K. Hattori, K. Kataoka, E. Takematsu, A. Ishii, F. Komori, and H. Daimon, J. Magn. Magn. Mater. 363, 158 (2014). 